\title{
Cognitive and Emotional Characteristics of Communication in Human-Human/Human-Agent Interaction
}

\author{
Yugo Hayashi and Kazuhisa Miwa \\ Graduate School of Information Science, Nagoya University, Furo-cho, Chikusa-ku, Nagoya, \\ 464--8601, Japan \\ \{hayashi, miwa\}@cog.human.nagoya-u.ac.jp
}

\begin{abstract}
A psychological experiment was conducted to capture the nature of Human-Human and Human-Agent Interactions where humans and computer agents coexist in a collaborative environment. Two factors were manipulated to investigate the influences of the 'schema' about and the 'actual partner' on the characteristics of communication. The first factor, expectation about the partner, was controlled by the experimenter's instruction, manipulating with which partner (human or computer agent) participants believed to be collaborating. The second factor, the actual partner, was controlled by manipulating with which partner (human or computer agent) participants actually collaborated. The results of the experiments suggest that the degree of the refinement of the conversation controlled as the actual partner factor affected the emotional and cognitive characteristics of communication; however the schema about the opponent only affected the emotional characteristics of communication.
\end{abstract}

Keywords: Collaboration, Human-Human Interaction, Human-Agent Interaction, Communication.

\section{Introduction}

Communication across different computers connected by the Internet continues to increase due to the development of computer network technologies. In such situations, research on technologies for supporting such collaboration using computer agents has appeared [6]. In the fields of Human Computer Interaction (HCI), there are studies focusing on the nature of humans and computer agents [3]. In our study, we conduct a psychological experiment to capture the nature of Human-Human Interaction (HHI) and Human-Agent Interaction (HAI) in a setting where humans and computer agents coexist.

In daily life, we make inferences and decisions about an opponent based on information received from it. For example, when he reacts politely, we may guess his character and attitudes through his reactions. This indicates that in communication the contents of conversation are important for determining the characteristics of communication. In contrast, in the initial stage of communication, information about an opponent is limited. Therefore, people rely on the related knowledge of an opponent obtained in advance and 
infer him based on this knowledge. Actually, social psychological research has indicated the importance of top-down processing based on the knowledge about the speaker such as schema and stereotypes in interpersonal cognition [1].

In communication through the Internet where we do not face an opponent directly, the above two perspectives, "actual conversation" and "schema," function as follows: (1) either the opponent is believed to be a human or computer agent (based on the schema), and (2) either the actual opponent's conversation is sophisticatedly adaptive or simply machine-like. We performed a psychological experiment in which the two factors were manipulated to investigate the characteristics of communication where humans and computer agents coexist.

\section{Method}

\subsection{Task}

We assume that the above interesting aspects of communication emerge remarkably in a situation where interpersonal conflicts emerge during collaboration. To establish such a situation, we used an experimental paradigm designed by Hayashi, Miwa, and Morita [2], in which two participants, each having a different perspective, communicate with each other. As shown in Figure 1, a stimulus is constructed where black and white unit squares are randomly arranged on a 6 - by 6-grid.

We call each surface comprised of black and white unit squares an 'object'. In Figure 1, there are a total of ten objects: five black and five white. This stimulus is displayed on either a black or white background. This stimulus is presented to one participant on a black background; to the other on a white background. Based on Gestalt psychological principles, the participants acquire a single perspective focusing on objects whose color is the opposite of the background color. Each participant informs the other of the sequence of the number of objects he/she perceives during the task (Figure 2). In the initial stage of the experiment, one experiences difficulty understanding the perspective of the other; with miscommunication, interpersonal conflict occurs, which the participants must resolve to complete the task.

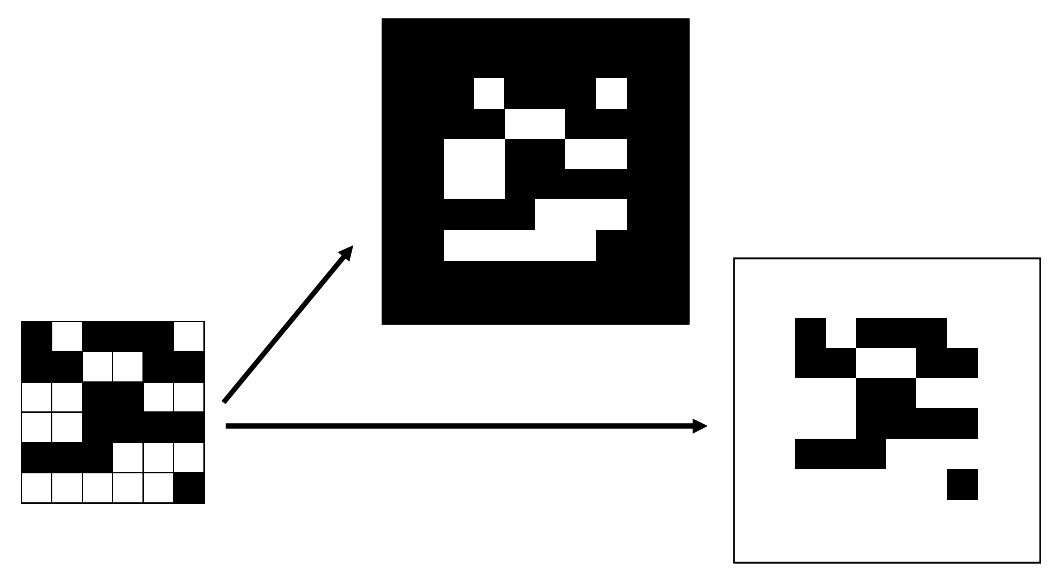

Fig. 1. Example of stimuli 


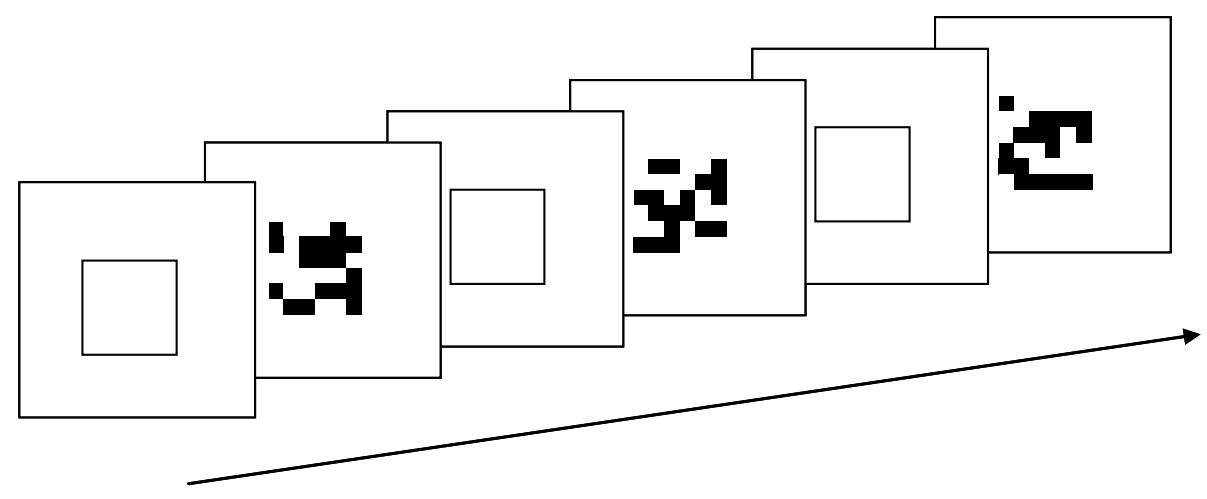

Fig. 2. Series of stimuli presentations

\subsection{Experimental System}

Figure 3 illustrates an example screen shot. The stimulus is presented in the center. Below it, there is a text field where the participants input their messages and receive their partner's messages. Just one sentence per trial is permitted, and at most 30 words are accepted. Buttons for changing the slides, sending messages, and terminating the experiment are placed at the bottom of the screen. We developed a natural language conversation agent whose responses are generated based on scripts. The agent is constructed to respond to the sentences input from the participants. This agent has mechanisms for conversation such as extracting keywords, activating scripts, and generating responses utilizing keywords drawn from the partner's sentences inputted.

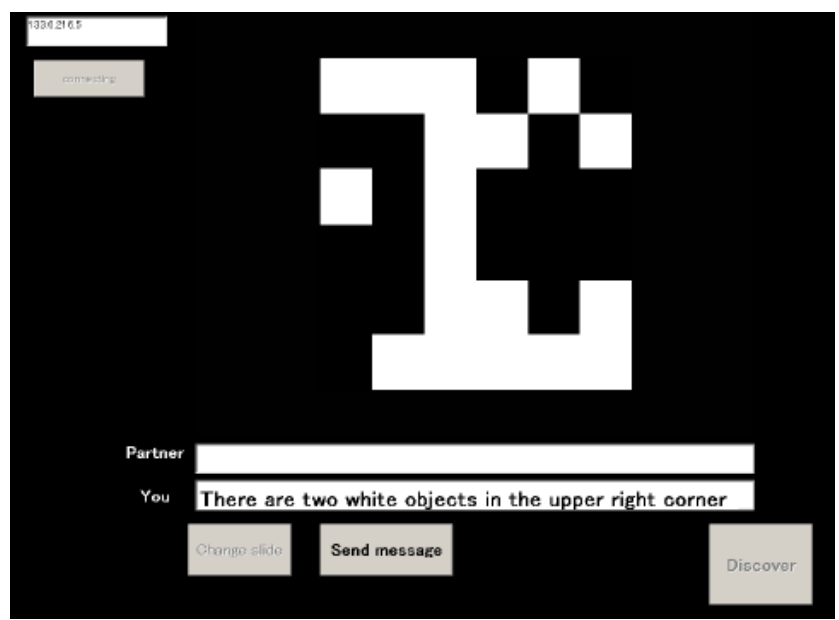

Fig. 3. Example screenshot 


\section{Experiment Design}

\subsection{Summary}

The experiment has a $2 \times 2$ between-subjects factorial design. The first factor was controlled by the experimenter who manipulated with which partner (human or computer agent) the participants believed themselves to be collaborating. This represents the manipulation for the schema about the opponent. The second factor was controlled by manipulating with which partner (human or computer agent) the participants actually collaborated. This represents the manipulation of the degree of refinement of the conversation as the actual partner. In the following, we use small letters to express characters in the first factor, human and agent, and capital letters to express characters in the second factor, HUMAN and AGENT. For example, in the agent/HUMAN condition, the participants were instructed that the collaborative partner was a computer program; however they conversed with a human partner (details are discussed below). We constructed four conditions: human (instruction)/HUMAN (actual partner), agent/HUMAN, human/AGENT, and agent/AGENT.

\subsection{Experiment Situation}

One hundred and three undergraduates participated in the experiment (male $=57$, female $=46, M$ age $=18.82$ years). They were set up to always speak first in the AGENT conditions where the participants conversed with the agent. On the other hand, the participants were set up to speak both first and second in the HUMAN conditions where the participants conversed with real people. Therefore, twice as many participants were assigned to the HUMAN conditions. Table 1 shows the number of participants assigned to each condition.

The experiment was performed in small groups consisting of eight to twelve participants. Two types of computers were set up in a laboratory: machines connected to the Internet by wireless LAN and those running independently of other computers. These computers were placed so that no participant could peek at other screens (Figure 4).

For manipulating the first factor, the participants were instructed that the collaborative partner was either: (1) someone in the room or (2) a program installed in the computer. For manipulating the second factor, the actual partner was controlled by assigning either: (1) a computer connected to someone in the room through wireless LAN or (2) one in which the conversation agent was running independently from others.

Table 1. Experimental design and number of assigned participants

Instruction

\begin{tabular}{c|c|c|c|}
\cline { 2 - 4 } Actual & & humans & agents \\
\cline { 2 - 4 } partner & HUMAN & 34 & 34 \\
\cline { 2 - 4 } & AGENT & 18 & 17 \\
\hline
\end{tabular}




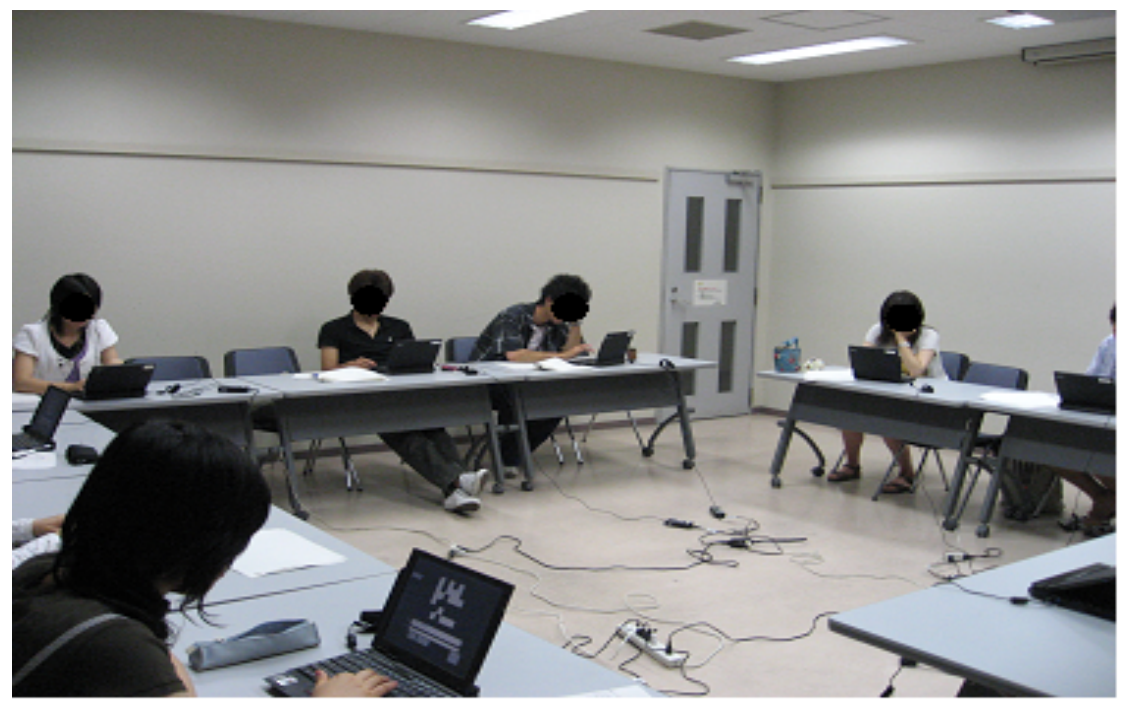

Fig. 4. Experimental situation

\subsection{Questionnaires}

In our study we utilized a questionnaire developed by Tsuduki and Kimura [4] answered by the participants after their conversations to solve the task were terminated. This questionnaire, which asks about the psychological characteristics of the media in communication, was comprised of 16 questions scored with a five-point scale. We classified the 16 questions into three measures. The first measure denotes the "interpersonal stress" factor consisting of five questions about such feelings as tension, severity, and fatigue. The second measure denotes the "affiliation emotion" factor consisting of eight questions about such feelings as friendliness to the opponent, ability to discuss personal matters, and happiness. The third measure denotes the "information propagation" factor consisting of three questions about such feelings as purpose and effectiveness in collecting information. In each of the three measures, the rating scores were totaled and divided by the number of subordinate questions. These rating scores (i.e., mean numbers) were used for analysis.

\section{Results}

Figure 5 indicates the results. The vertical axis represents the mean value of the ratings, and the horizontal axis represents each measure. For participants who talked first, a 2 × 2 ANOVA was conducted on each measure with the factor of instruction (human vs. agent) and the factor of actual partner (HUMAN vs. AGENT) as a between-subject factor. For participants who talked second, a t-test was conducted on each measure. These participants were assigned only to two conditions: human/HUMAN and agent/HUMAN; therefore, only the effect of the instruction factor was examined. 


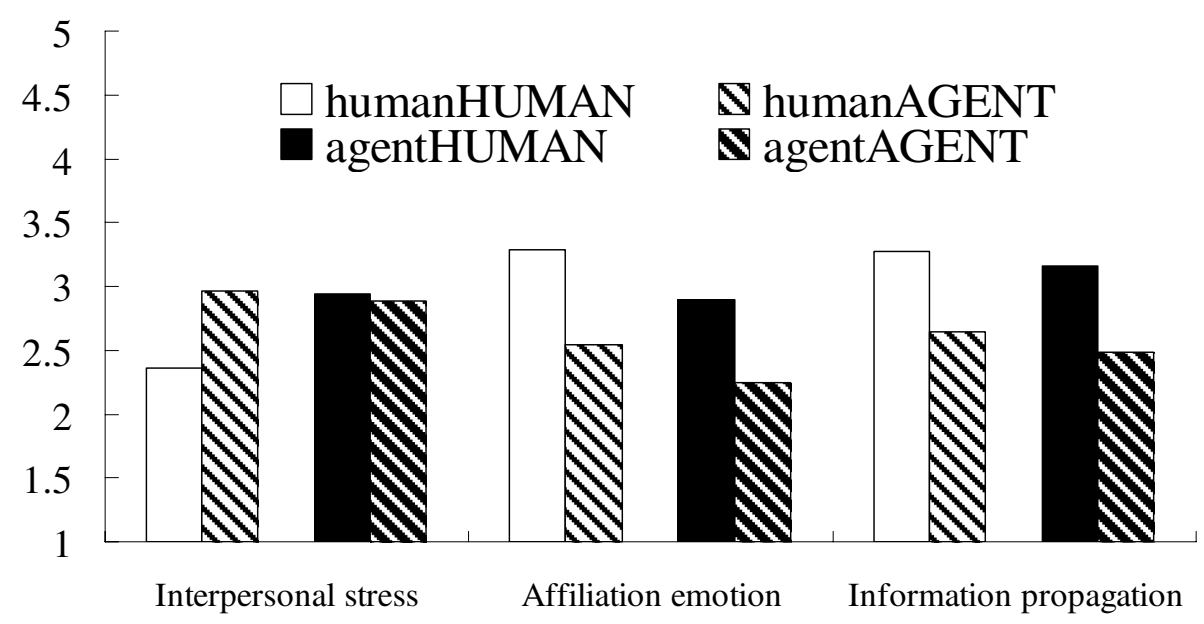

(a) Participants who talked first

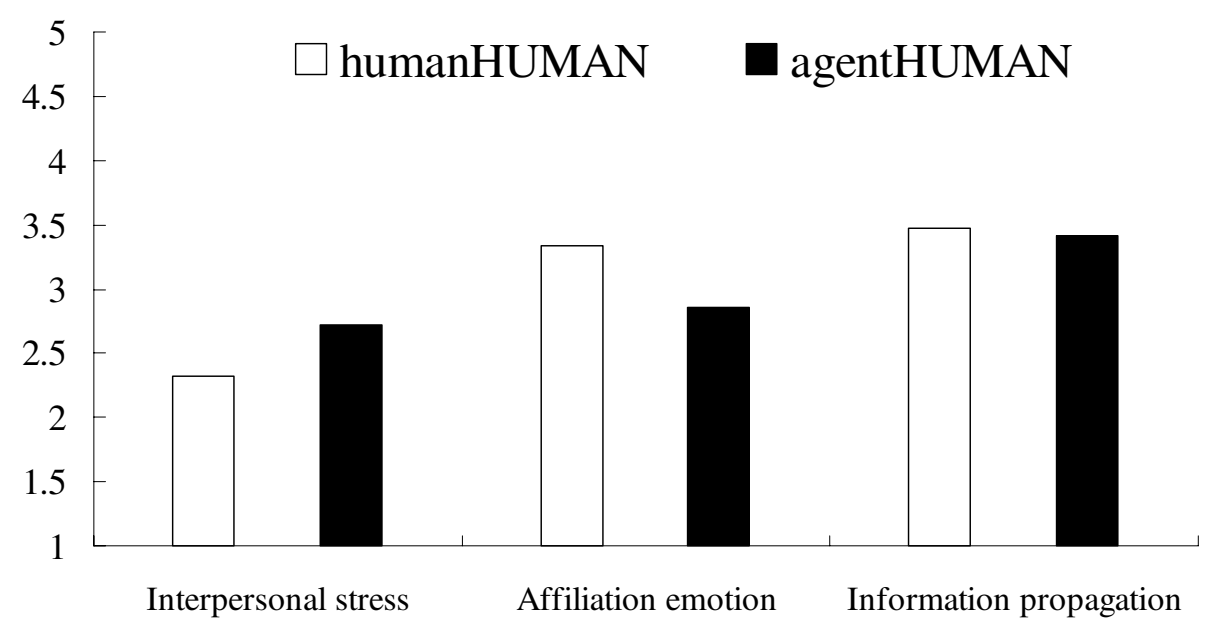

(b) Participants who talked second

Fig. 5. Questionnaires

\subsection{Interpersonal Stress}

For participants who talked first, interaction on interpersonal stress was significant $(\mathrm{F}(1,65)=7.34, \mathrm{p}<.01)$. An analysis of the simple main effect was conducted. Focusing on the actual partner factor, the rating score of the human condition was significantly higher than the agent condition in the HUMAN condition $(\mathrm{p}<.01)$. On the other hand, the rating score was not significantly different in the AGENT condition ( $\mathrm{p}=.63)$. Focusing on the instruction factor, the rating score in the AGENT condition was significantly higher than the HUMAN condition in the human condition $(\mathrm{p}<.01)$. On the other hand, the rating score was not significantly different in the agent condition 
$(\mathrm{p}<.01)$. In addition, there was a main effect of both factors of instruction and actual partner $(\mathrm{F}(1,65)=4.07, \mathrm{p}<.05 ; \mathrm{F}(1,65)=4.96, \mathrm{p}<.05)$. For participants who talked second, the rating score in the agent/HUMAN condition was significantly higher than in the human/HUMAN condition $(\mathrm{F}(1,32)=4.25, \mathrm{p}<.05)$.

\subsection{Affiliation Emotion}

For participants who talked first, the interaction of affiliation emotion was not significant $(\mathrm{F}(1,65)=0.06, \mathrm{p}=.8)$. There was a marginal main effect of the factor of instruction $(\mathrm{F}(1,65)=3.66, \mathrm{p}=.06)$, and the rating score of the human condition was marginally higher than the agent condition. There was a main effect of the factor of actual partner $(\mathrm{F}(1,65)=15.12, \mathrm{p}<.01)$, and the rating score of the HUMAN condition was significantly higher than the AGENT condition. For participants who talked second, the rating score of the human/HUMAN condition was significantly higher than the agent/HUMAN condition $(\mathrm{F}(1,32)=6.70, \mathrm{p}<.05)$.

\subsection{Information Propagation}

For participants who talked first, the interaction of information propagation was not significant $(\mathrm{F}(1,65)=0.01, \mathrm{p}=.92)$. There was not a main effect of the factor of instruction $(\mathrm{F}(1,65)=0.48, \mathrm{p}=.49)$. There was a significant main effect of the factor of actual partner $(\mathrm{F}(1,65)=10.49, \mathrm{p}<.01)$, and the rating score of the HUMAN condition was significantly higher than the AGENT condition. For participants who talked second, the rating score was not statistically significant $(\mathrm{F}(1,32)=0.10, \mathrm{p}=.75)$.

\subsection{Summary}

Table 2 summarizes the statistical results. The asterisk represents a significant difference, the plus sign represents a marginal difference, and the minus sign represents no differences.

Table 2. Summary of results

\begin{tabular}{|l|c|c|c|c|}
\hline \multirow{2}{*}{} & \multicolumn{2}{|c|}{ Talking first } & \multicolumn{2}{c|}{ Talking second } \\
\cline { 2 - 5 } & Instruction & Actual partner & Instruction & Actual partner \\
\hline Interpersonal stress & $*$ & $*$ & $*$ & \\
\hline Affiliation emotion & + & $*$ & $*$ & \\
\hline Information propagation & - & $*$ & - & \\
\hline
\end{tabular}

\section{Discussion and Conclusion}

We assumed that the interpersonal stress and affiliation emotion scores are related to the 'emotional' features of communication, whereas the information propagation score is related to the 'cognitive' features of communication. The overall results of the experiments suggest the following: (1) the degree of the refinement of the conversation 
controlled as the actual partner factor affected the emotional and cognitive characteristics of communication, and (2) the schema about the opponent only affected the emotional characteristics of communication.

In Yamamoto, Matsui, Hiraki, Umeda, and Anzai [5], the participants played Shiritori, a popular Japanese word game, with a partner by computer. Even though the actual identity of their partners was a computer agent, the participants, who were informed that they were facing a human player, gave significantly higher pleasure ratings than those who were informed that they were facing a computer player. Pleasure ratings are representative emotional measures. Therefore, the result of the preceding study is consistent with our finding: i.e., the instruction effect, relying on the participants' schema about their partners, appears in the emotional characteristics of communication.

However, as a whole, the effect of the actual partner factor was dominant, and it was detected in all measures. This contradicts the findings of a study with a simple computer program called ELIZA, a counselor-like agent program [7]. Even though the responses were very simple, people felt empathy throughout their interaction with ELIZA. This study indicates that the correlation is not simple between the elaboration of a partner's conversation and the quality of interaction. What caused the difference? In the preceding study, the participants conversed with the computer without any specific task goals. However, in our study, a relatively complicated situation was given to the participants, who had to find a rule as a task goal. These characteristics of our task probably caused the difference: i.e., the actual partner factor was dominant in our study, while in the previous study the effect of actual partner was limited.

\section{References}

1. Fisk, T.S., Taylor, E.S.: Social cognition. McGraw-Hill Education, New York (1991)

2. Hayashi, Y., Miwa, K., Morita, J.: A laboratory study on distributed problem solving by taking different perspectives. In: Proceedings of the 28th Annual Conference of the Cognitive Science Society, pp. 333-338 (2006)

3. Parise, S., Kiesler, S., Sproull, L., Waters, K.: Cooperating with life-like interface agents. Computers in Human Behavior 70, 123-142 (1999)

4. Ye, Y., Churchill, E.F.: Agent supported cooperative work. Kluwer Academic Publisher, Dordrecht (2003)

5. Tsuduki, T., Kimura, Y.: Characteristics of media communication of college students: comparing face to face, mobile phone, mobile mail, and electronic mail. Applied sociology studies 42, 15-24 (2000) (in Japanese)

6. Yamamoto, Y., Matui, T., Hiraki, K., Umeda, S., Anzai, Y.: Interaction with a computer system:-a study of factors for pleasant interaction. Cognitive Studies 1, 107-120 (1994) (in Japanese)

7. Weizenbaum, J.: A computer program for the study of natural language communication between man and machine. Communications of the Association for Computing Machinery 9 , 36-45 (1966) 